\title{
Zur Frage nach der Homologie der Blepharoplasten.
}

\author{
Von S. Ikeno.
}

Seit der Publikation meiner Untersuchungen über die Spermatogenese von Marchantia polymorpha ${ }^{1}$ ) sind verschiedene Arbeiten über dasselbe Thema erschienen, sowohl was Lebermoose als auch andere Pflanzengruppen betrifft, was mich veranlaßt, in dem vorliegenden Aufsatz auf diese Arbeiten-zurückzukommen und meine Ansicht über die Homologie der Blepharoplasten noch mehr zu präzisieren.

Eine der neuesten Publikationen ist eine kurze Mitteilung Miyakes über die Spermatogenese bei verschiedenen Lebermoosgattungen, wie Marchantia, Fegatella, Pellia, Makinoa, Aneura ${ }^{2}$ ). In keiner dieser Gattungen konnte er bei den sukzessiven Kernteilungen in den Antheridien dié Zentrosomen nachweisen und deshalb stellte er meine positive Angabe über Marchantia in Zweifel. Da man daher vielleicht glauben könnte, daß meine diesbezügliche Beobachtung auf einem Irrtum beruhe, so habe ich in einem kleinen Aufsatz gezeigt, daß meine Angabe vollständig richtig ist und die negativen Resultate Miyakes auf einer der drei folgenden Möglichkeiten beruhen könnten, entweder auf Verwendung schlechten Materials, oder auf unzureichender Behandlung desselben, oder auf Übersehen der in Frage stehenden sehr kleinen Körperchen ${ }^{3}$ ). Diejenigen Leser jedoch, welche noch weitere Bestätigung verlangen, seien auf die in der neuesten Zeit erschienene Arbeit Lewis über einige Riccia-Arten hingewiesen ${ }^{4}$ ). Dieser Forscher studierte unter anderem die Spermatogenese dieser Lebermoosarten und fand dabei solche Körperchen, welche dieselbe Stellung bei dem Zellkerne einnehmen und dasselbe Verhalten zeigen, wie diejenigen, welche ich

1) Die Spermatogenese von Marchantia polymorpha. Beih. z. Bot. Zentralbl. 1903, Bd. XV.

2) On the centrosome of Hepaticae. The Bot. Magazine, Tokio 1905, Vol. XIX, No. 224.

3) Are the centrosomes in the antheridial cells of Marchantia polymorpha imaginary? Ibid., No. 225.

4) The Embryology and development of Riccia lutescens and Riccia crystallina. The Bot. Gaz. 1906, Vol. XLI. 
bei Marchantia fand und als Zentrosomen bezeichnete. Nach seiner Angabe $^{1}$ ) nämlich über die Zellkerne der Antheridien von Riccia sitzen diese Körperchen an den entgegengesetzten Polen der Kernspindel, verschwinden beim Schluß jeder Kernteilung, ausgenommen der letzten (sog. „diagonalen Teilung“), wobei sie verbleiben und die blepharoplastische Funktion spielen. Lewis nannte diese Körperchen „Zentrosomartige Körper" und scheint zu der Homologisierung derselben mit Zentrosomen nicht geneigt zu sein ${ }^{2}$ ), allein kein mit diesen Dingen näher Vertrauter wird die Identität derselben mit meinen „Zentrosomen“ bezweifeln. Diese Arbeit Lewis, welche, wie oben gezeigt, mit meinem Befunde an Marchantia fast völlig übereinstimmt, mag deshalb als eine schöne Bestätigung meiner in Frage stehenden Angabe dienen.

Bei seinen Studien über Fegatella conica fand Bolleter zwar keine Zentrosomen an den Spindelpolen bei den Antheridien-Kernteilungen ${ }^{3}$ ), da aber seine Beobachtung mit durch Alkohol fixiertem Material ausgeführt wurde, so gibt er selbst $\mathrm{zu}^{4}$ ), daß die Zentrosomen wirklich vorhanden gewesen sein können und nur an den nach den modernen Fixierungsmethoden behandelten Präparaten wahrzunehmen sind. Die negative Angabe Miyakes über dieselbe Art wird vielleicht einer Nachprüfung bedürfen.

Fast gleichzeitig mit der Lewisschen Arbeit erschien eine Studie Humphreys über Fossombronia ${ }^{5}$ ). Danach erscheint der Blepharoplast zuerst im Zytoplasma der Spermatiden, wo er sofort seine blepharoplastische Rolle spielt $\left.{ }^{6}\right)$. Somit verhält sich hier der Blepharoplast nach einer von zwei Möglichkeiten, welche ich im Jahre 1904 vermutungsweise ausgesprochen habe, ,sie treten nämlich niemals während der spermatogenetischen Kernteilungen auf, sondern sie entstehen zuerst in der Spermatide, wo sie sofort die blepharoplastische Funktion ausüben" "7). Wie ich dort gezeigt habe ${ }^{8}$ ), sind bei Pellia epiphylla und Makinoa crispata keine Zentrosomen bei den Kernteilungen in den Antheridien

1) 1. c. pag. 109 .

2) 1. c. pag. 132 .

3) Fegatella conica (L.) Corda. Beih. z. Bot. Zentralbl. 1905, Bd. XVIII, Erste Abteil.

4) l. c. pag. 349 .

5) The development of Fossombronia longiseta, Aust. Ann. of Bot. 1906, Vol. XX, No. 77.

6) 1. c. pag. $96 \mathrm{ff}$.

7) Blepharoplasten im Pflanzenreich. Biol. Zentralbl. 1904, Bd. XXIV, pag. 219, Fußnote.

8) 1. c. pag. 219 . 
nachzuweisen und so ist es höchst wahrscheinlich, daß das Verhalten der Blepharoplasten dieser zwei Arten mit dem übereinstimmt, was bei Fossombronia geschieht ${ }^{1}$ ).

Nach alledem komme ich bezüglich der Blepharoplastenfrage der Lebermoose zum folgenden SchluB, welcher mit dem von mir im Jahre 1904 ausgesprochenen ${ }^{2}$ ) fast völlig übereinstimmt und lautet: Bei den unteren Lebermoosen (z. B. Marchantiales, wie Marchantia, Riccia), spielen die Zentrosomen sowohl ihre normale als blepharoplastische Funktion; im Laufe der Phylogenie dieser Pflanzengruppe büßten sie allmählich die erstere Funktion, um nun lediglich für letztere spezialisiert zu werden, womit nach dem bekannten Lamarckschen Prinzip das allmähliche Verschwinden dieser Körperchen an den Stellen, für die sie nicht mehr nötig waren, stattgefunden hat. Es ist deshalb nicht unmöglich, daß man solche Lebermoose auffinden könnte, bei denen die Zentrosomen, trotzdem sie die nämliche Stelle einnehmen wie die gewöhnlichen, doch schon die normale Rolle eingebüßt haben.

- Mottier, in seinen Studien über die Spermatogenese von Chara ${ }^{3}$ ), stimmt mit mir überein betreffend die zentrosomatische Natur derjenigen Körperchen, welche ich in jungen Zellgenerationen der Antheridien aufgefunden und als Zentrosomen gedeutet habe ${ }^{4}$ ), allein die von mir angegebene Tatsache, daß das Zentrosom bei der diagonalen Teilung in den Antheridien verbleibt, wird von ihm in Zweifel gestellt, weil diejenigen Körperchen, welche als Blepharoplasten fungieren und diejenigen, welche ich bei der Kernteilung an den Spindelpolen wahrgenommen hatte und als Zentrosomen deutete, trotz ihres gleichartigen Aussehens ganz verschiedene Dinge sein könnten $\left.{ }^{5}\right)$. Darin kann ich jedoch diesem Forscher ,nicht zustimmen und ich glaube noch jetzt, daß die nämlichen Körper, welche bei der diagonalen Teilung als Zentrosom fungiert hatten, verbleiben und als Zilienbildner dienen. Daß diese Anschauungen weit wahrscheinlicher sind als die Mottiers, wird für denjenigen klar werden, welcher meine Angabe mit der Jahnschen Untersuchung an einigen

1) Es war meine Absicht, sofort nach dem Abschluß meiner Untersuchungen von Marchantia meine Studien an Pellia, Makinoa usw. fortzusetzen, allein wegen anderer Beschäftigungen konnte ich bisher diese Arbeit noch nicht ausführen. Doch dürften weitere Studien kaum mehr nötig sein, nachdem die schöne Arbeit $\mathrm{Hum}$ phreys erschienen ist.

2) 1. c. pag. $219-220$. Vol. XVIII.

3) The development of the spermatozoid in Chara. Ann. of Bot. 1904,

4) 1. c. pag. 250 .

5) l. c. pag. 252 . 
Myxomyceten ${ }^{1}$ ) vergleichen will. Danach kann man bei der Kern- und Zellteilung der Schwärmer an den beiden Spindelpolen je ein Zentrosom wahrnehmen und während diese an den Polen sitzen, sieht man eine Zilie daraus hervorwachsen, was mit der Angabe Henneguys über die Spermatozyten einiger Schmetterlingsarten ${ }^{2}$ ) übereinstimmt. Da in diesen Fällen die aus den Zilien noch an den Spindelpolen sitzenden Zentrosomen hervorsprossen, ist aus naheliegenden Gründen die Identität der Zentrosomen und Blepharoplasten nicht mehr zu leugnen und es kann kaum mehr zweifelhaft sein, daß auch bei Marchantia beide Gebilde, Zentrosom und Blepharoplast, die nämlichen sind.

- Nach Mottier stammen die Blepharoplasten bei den Spermatiden von Chara aus der Hautschicht des Zytoplasmas ${ }^{3}$ ); wenn diese Angabe des amerikanischen Forschers sich bestätigt, so dürften vielleicht die von Strasburger untersuchten Blepharoplasten an den Schwärmsporen einiger Chlorophyceen ${ }^{4}$ ) zu derselben Kategorie gehören. Nach Schaudinn gehen dieselben bei einigen Flagellatengattungen aus ganzen Kernen hervor ${ }^{5}$ ). Ich bin noch nicht im Besitz dieser Arbeit Schaudinns und deshalb kann ich nicht selbst die Richtigkeit seiner Angabe beurteilen. Allein wenn man diese Angaben Mottiers, Strasburgers und Schaudinns für richtig hält, dann wären alle sog. „Blepharoplasten“ keine morphologisch einheitlichen Gebilde, wie ich auch zuerst zu glauben geneigt war ${ }^{6}$ ), und man könnte dann die drei folgenden Kategorien unterscheiden :

1) Myxomycetenstudien. 3. Kernteilung und Geißelbildung bei den Schwärmern von Stemonitis flaccida Lister. Ber. d. Deutschen Bot. Gesellschaft 1904, Bd. XXII.

2) Wilson, The cell in development and inheritance, second edition, New York 1900, pag. 357, Fig. 167, obere Figur.

3) 1. c. - Mottier hat die Entwicklung der Spermatozoiden aus den Spermatiden genau untersucht und kam zu dem Schlusse, daß der Blepharoplast aus der Hautschicht der Spermatide stammt. Allein meiner Ansicht nach wäre es vielleicht nicht unmöglich, daß durch die Untersuchung der jüngeren Entwicklungsstadien, als die von diesem Forscher studierten, die Abstammung dieser ,plasmodermalen“ Gebilde aus den Zentrosomen oder ähnlichen Körpern festgestellt werden könnte.

4) Über Reduktionsteilung, Spindelbildung, Zentrosomen und Zilienbildner im Pflanzenreich. Histolog. Beitr. 1900, Heft VI.

5) Generations- und Wirtswechsel bei Trypanosoma und Spirochaete. (Vorläufige Mitteilung.) Arbeiten aus dem kaiserlichen Gesundheitsamte 1904, Bd. XX, Heft 3. Ich kenne diese Arbeit nur aus dem Zitate in Jahns Aufsatz 1. c.

6) Die gleiche Ansicht wurde auch von Jahn ausgesprochen, l. c. pag. 91. 
1. Zentrosomatische Blepharoplasten sind solche, welche entweder onto- oder phylogenetisch zentrosomatischen Ursprungs sind; soweit untersucht, gehören fast alle Blepharoblasten zu dieser Kategorie. Myxomyceten, Lebermoose, Gefäßkryptogamen, Gymnospermen.

2. Plasmodermale Blepharoplasten. Chara, einige Chlorophyceen.

3. Karyo- oder Kern-Blepharoplasten. Nur bei einigen Flagellatengattungen. 